\title{
Analyzing the Quenchable Iron Pool in Murine Macrophages by Flow Cytometry
}

\author{
Michael Riedelberger and Karl Kuchler*
}

Medical University of Vienna, Center for Medical Biochemistry, Max Perutz Labs Vienna, Campus Vienna Biocenter, A-1030 Vienna, Austria

*For correspondence: karl.kuchler@meduniwien.ac.at

[Abstract] Tissue-resident macrophages are pivotal for a tightly-regulated iron metabolism at a cellular and systemic level, since subtle iron alterations increase the susceptibility for microbial infections or drive multiple diseases. However, research on cellular iron homeostasis in macrophages remains challenging due to the limited amount of available methods using radioactive ${ }^{59} \mathrm{Fe}$ isotopes or strong iron chelators, which might be inapplicable in certain experimental settings. This protocol describes the analysis of the quenchable iron pool (QIP) in macrophages by loading these cells with exogenous iron-complexes. Thereby, the cytoplasmic iron pool can be determined, since the iron uptake ability of macrophages inversely correlates with intracellular iron levels. Thus, this assay enables the accurate analysis of even minor alterations in cytoplasmic iron fluxes and is applicable in almost every laboratory environment. In addition, the protocol can also be adopted for other immune cell types in vitro and in vivo.

Keywords: Quenchable iron pool, Labile iron pool, Cytoplasm, Macrophage, Flow cytometry, Calcein-AM, 8-hydroxyquinoline, FeHQ

[Background] Owing to their central regulatory functions for systemic iron metabolism, macrophages in multiple tissues swiftly alter their intracellular iron levels upon encounter of diverse stimuli during microbial infections and diseases (Nairz et al., 2017). Of note, the majority of cellular iron ions is bound by the major iron storage protein Ferritin within the cytoplasm or is localized to compartments like mitochondria or lysosomes (Ma et al., 2015; Soares and Hamza, 2016). However, the labile iron pool represents an unbound fraction of cytoplasmic iron, which is metabolically accessible and pivotal for cellular iron homeostasis and metabolism (Cabantchik, 2014). Interestingly, subtle concentration changes within this macrophage iron pool can greatly impact the outcome of infections, especially with intracellular pathogens (Ganz and Nemeth, 2015).

However, these intracellular iron fluxes in the nanomolar to millimolar range are highly dynamic and are additionally regulated in a spatiotemporal manner, providing a significant challenge for accurate experimental quantification (Cabantchik, 2014; Ma et al., 2015). Methods for iron import/export studies, using radioactive ${ }^{59} \mathrm{Fe}$ isotopes, are highly sensitive and specific, but are quite limited in their application for the broad interested scientific community owing to safety issues and governmental regulations. Further, quantification of chelated iron by spectroscopy is hampered by either physical cell disruption, low sensitivity or the large amounts of required biological materials. 
To address these experimental challenges, Epsztejn et al. described a Calcein-AM-based method to analyze the labile iron pool (Epsztejn et al., 1997). When Calcein-AM is taken up by metabolically active cells, this acetoxy-methyl-ester is cleaved by intracellular esterases, releasing the iron-specific fluorescent dye Calcein exclusively into the cytoplasm (Ma et al., 2015). Thereby, Calcein fluorescence is quenched upon binding to cytoplasmic iron ions (Figure 1). Further, this method was refined for analysis by flow cytometry (Prus and Fibach, 2008). In general, these protocols rely on the usage of strong iron chelators to sequester cytoplasmic iron ions for subsequent Calcein fluorescence rescue. However, some iron chelators are commercially unavailable (e.g., salicylaldehyde isonicotinoyl hydrazone $=\mathrm{SIH}$ ) or can have diminished chelating effects under specific experimental conditions.

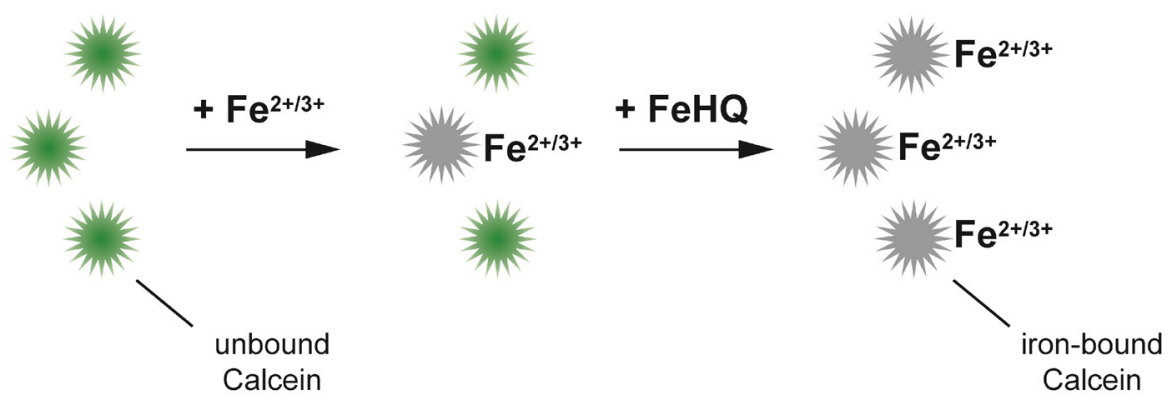

Figure 1. Quenching of cellular Calcein fluorescence upon binding of iron ions. Calcein fluoresces in the unbound state but gets quenched during binding by accessible iron ions within the cytoplasm. Addition of exogenous iron upon treatment with $\mathrm{FeCl}_{2} / 8$-hydroxyquinoline (FeHQ) complexes further quenches Calcein fluorescence.

Thus, in order to increase practical applicability, this Calcein-AM-based assay was modified by analyzing the quenchable iron pool (QIP) instead (Du et al., 2015; Siegert et al., 2015). Since Calcein shows reduced fluorescence in the iron-bound state, its fluorescence gets additionally quenched when exogenous iron is swiftly delivered into the cytoplasm by the addition of a $\mathrm{FeCl}_{2} / 8$-hydroxyquinoline (FeHQ) complex (Figure 1) (Prachayasittikul et al., 2013; Ma et al., 2015; Chobot et al., 2018). Hence, the difference in cellular Calcein fluorescence before and after FeHQ addition represents the QIP (Figure 2A). Of note, the size of the unbound, cytoplasmic iron pool inversely correlates with the ability of a given cell to take up iron ions. Thus, iron-starved cells can substantially take up more exogenous $\mathrm{Fe}$ (upon FeHQ addition) than iron-saturated cells (Figure 2B). Therefore, cells with minor cytoplasmic iron pools exhibit higher QIPs when compared to iron-loaded cells with reduced QIPs (Figure 2A).

In conclusion, QIP analysis by flow cytometry represents a powerful tool to accurately determine alterations in cytoplasmic iron levels upon diverse cellular stimuli. Further, this protocol can be established easily in almost every laboratory environment and can also be applied for the investigation of iron homeostasis in other immune cell types in vitro and in vivo. 
A

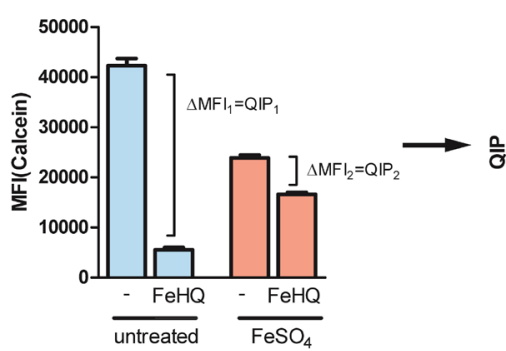

B

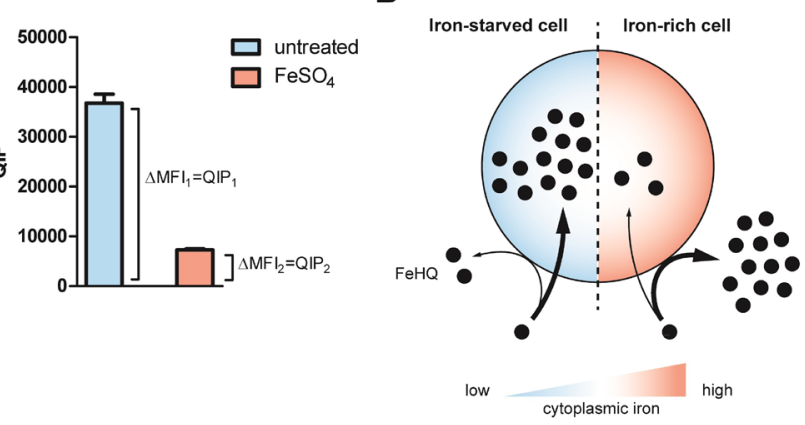

Figure 2. Theoretical concept of QIP analysis. A. Schematic representation of QIP calculation. Primary bone marrow-derived macrophages (BMDMs) were left untreated or treated with $50 \mu \mathrm{M}$ $\mathrm{FeSO}_{4}$ for $17 \mathrm{~h}$ and the MFI (Calcein) was determined in naive and FeHQ-challenged BMDMs. The QIP at the respective condition is calculated as the MFI difference. B. The cytoplasmic levels of unbound iron inversely correlate with the cellular iron uptake ability. Thus, cells with low cytoplasmic iron levels can take up more exogenous iron (upon FeHQ treatment), than already iron-saturated cells. MFI, median fluorescence intensity.

\section{Materials and Reagents}

1. Pipette tips

2. $1.5 \mathrm{ml}$ reaction tubes

3. $50 \mathrm{ml}$ conical centrifuge tubes (Starlab, catalog number: E1450-0200)

4. $15 \mathrm{ml}$ conical centrifuge tubes (Starlab, catalog number: E1415-0200)

5. Tissue papers (Tork, catalog number: 290163)

6. $5 \mathrm{ml}$ serological pipettes (Starlab, catalog number: E4860-0005)

7. $10 \mathrm{ml}$ serological pipettes (Starlab, catalog number: E4860-0010)

8. $5 \mathrm{ml}$ round-bottom FACS tubes, for example Falcon ${ }^{\mathrm{TM}}$ Round-Bottom Polystyrene Tubes (Thermo Fisher Scientific, Falcon ${ }^{\mathrm{TM}}$, catalog number: 352054 )

9. 6-well CytoOne ${ }^{\circledR}$ Plates, tissue culture-treated (Starlab, catalog number: CC7682-7506)

10. 24-well CytoOne ${ }^{\circledR}$ Plates, tissue culture-treated (Starlab, catalog number: CC7682-7524)

11. $10 \mathrm{~cm}$ round CytoOne ${ }^{\circledR}$ Dish, tissue culture-treated (Starlab, catalog number CC7682-3394)

12. T-150 CytoOne ${ }^{\circledR}$ Flask, tissue culture-treated, vented (Starlab, catalog number: CC7682-4815)

13. Thermo Scientific ${ }^{T M}$ Sterilin $^{T M} 100 \mathrm{~mm}$ Square Petri Dishes (Thermo Fisher Scientific, Thermo Scientific ${ }^{\top M}$, catalog number: 11349273 )

14. Natural-rubber scraper (Deutsch \& Neumann, catalog number: 2260001)

15. Rapid-Flow ${ }^{\mathrm{TM}}$ sterile disposable filter unit with cellulose nitrate-membrane: $0.2 \mu \mathrm{m}$ pore size (Thermo Scientific ${ }^{\mathrm{TM}}$, Nalgene ${ }^{\mathrm{TM}}$, catalog number: 10261741)

16. Primary bone marrow-derived macrophages (BMDMs) from C57BL/6J mice: For BMDM cultivation in general, please refer to Bourgeois et al., 2009

17. L929 cell line (ATCC, catalog number: CCL-1) 
18. Purified anti-mouse CD16/32 antibody (BioLegend, catalog number: 101302)

19. FITC anti-mouse/human CD11b antibody (BioLegend, catalog number: 101205)

20. PE anti-mouse $F 4 / 80$ antibody (BioLegend, catalog number: 123110)

21. Ultra-pure, high quality water, suitable for trace metal analysis (Sigma-Aldrich, catalog number: 14211)

22. DMEM (Thermo Fisher Scientific, Gibco ${ }^{\mathrm{TM}}$, catalog number: 11584486)

23. Fetal calf serum (FCS) (Sigma-Aldrich, catalog number: F7524)

24. Penicillin/Streptomycin (Sigma-Aldrich, catalog number: P4333)

25. Phosphate-buffered saline (PBS), $\mathrm{Ca}^{2+}$ and $\mathrm{Mg}^{2+}$ free, liquid (Sigma-Aldrich, catalog number: D8537)

26. $\mathrm{FeCl}_{2} \cdot 4 \mathrm{H}_{2} \mathrm{O}$ (Sigma-Aldrich, catalog number: 220299)

27. 8-hydroxyquinoline (Sigma-Aldrich, catalog number: H6878)

28. Calcein-AM (BioLegend, catalog number: 425201)

29. Dimethyl sulfoxide (DMSO) (Sigma-Aldrich, catalog number: D2438)

30. Trypsin solution 10x (Sigma-Aldrich, catalog number: 59427C)

31. EDTA disodium salt dihydrate (AppliChem, catalog number: 131669.1211)

32. Bovine serum albumin (BSA) (Sigma-Aldrich, catalog number: A2153)

33. Complete DMEM (see Recipes)

34. L929-conditioned medium (see Recipes)

35. BMDM medium (see Recipes)

36. Stock solution preparation of Calcein-AM, $\mathrm{FeCl}_{2}$ and 8-hydroxyquinoline (see Recipes)

37. Calcein-AM staining solution (see Recipes)

38. FeHQ solution (see Recipes)

39. Trypsin solution (see Recipes)

40. FACS buffer (see Recipes)

\section{Equipment}

1. Pipettes, for example Pipetman model (Gilson)

2. Motorized single-channel pipette (Gilson, Pipetman ${ }^{\circledR}$ Concept 100-1,200 $\mu$ l)

3. Combined orbital linear shaking water bath (Grant Instruments, model: OLS200)

4. Laminar flow hood suitable for cell culture, for example Microbiological Safety Cabinets (Szabo-Scandic, SafeFAST Premium 212)

5. $\mathrm{CO}_{2}$-incubator (Binder, model: $\mathrm{CB}$ 170)

6. Automated cell counter, for example CASY cell counter \& analyzer (Roche, model: TTC-2KA-2037)

7. Refrigerated centrifuge (Hettich, model: Rotina 38R)

8. Refrigerated bench top centrifuge (Peqlab, PerfectSpin 24R Refrigerated Microcentrifuge) 
9. Flow cytometer equipped with appropriate lasers and filters for the detection of Calcein fluorescence, for example BD LSRFortessa ${ }^{\mathrm{TM}}$ (BD Biosciences)

\section{Software}

1. BD FACSDiva Software (BD Biosciences, Version: 8.0.1)

2. FlowJo Software (FlowJo, Version: 7.6.5)

\section{Procedure}

A. BMDM harvest and preparation

1. For differentiation of primary bone marrow-derived macrophages (BMDMs), cultivate murine bone marrow cells (isolated from one tibia and femur) in BMDM medium and $10 \mathrm{~cm}$ square tissue culture dishes for 10 days at $37{ }^{\circ} \mathrm{C}, 5 \% \mathrm{CO}_{2}$.

Note: For BMDM cultivation in general, please refer to Bourgeois et al. (2009).

2. Harvest BMDMs from $10 \mathrm{~cm}$ square dishes by gently scraping with a natural-rubber scraper $24 \mathrm{~h}$ before the experiment on Day 10 of cultivation and centrifuge at $300 \times \mathrm{g}, 20^{\circ} \mathrm{C}$ for $6.5 \mathrm{~min}$.

3. Aspirate medium and resuspend the BMDM pellet in $1 \mathrm{ml} \mathrm{BMDM} \mathrm{medium.}$

4. Accurately determine the BMDM cell concentration by using an automated cell counter.

5. Transfer $2.5 \times 10^{5}$ BMDMs into a 24-well plate, supplied with a total volume of $300 \mu \mathrm{l} \mathrm{BMDM}$ medium.

Note: Per every experimental condition, at least 3 technical replicates each for untreated and

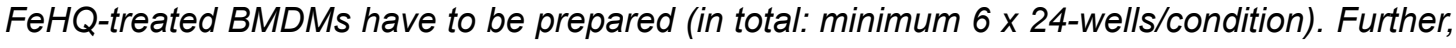
include one replicate of untreated BMDMs as unstained control for flow cytometry analysis.

6. Incubate BMDMs overnight at $37^{\circ} \mathrm{C}, 5 \% \mathrm{CO}_{2}$.

B. BMDM stimulation

1. Stimulate BMDMs with the respective reagent (e.g., LPS, cytokines, bacterial or fungal pathogens) under the desired condition.

2. Incubate BMDMs at $37^{\circ} \mathrm{C}, 5 \% \mathrm{CO}_{2}$.

C. BMDM staining and preparation for flow cytometry analysis

1. Thirty minutes before the end of BMDM stimulation, warm PBS to $37^{\circ} \mathrm{C}$ in a water bath, freshly prepare FeHQ solution in sufficient amounts and adjust all required materials for subsequent BMDM staining procedure.

2. Immediately before the end of the BMDM experiment, prepare the Calcein-AM staining solution and store it in the dark. 
Note: Calcein-AM is highly susceptible for hydrolysis in aqueous buffer solutions. Therefore, to ensure maximal BMDM staining efficiency, work as quick and as accurate as possible from this step on.

3. Remove the BMDM medium by quickly tilting the 24-well plate onto a stack of tissue papers.

4. Wash BMDMs $3 x$ with $300 \mu$ pre-warmed PBS and remove the wash solution by quickly tilting the 24-well plate onto the used stack of tissue papers.

Note: Completely remove FCS-containing cell culture medium, since FCS residuals alter Calcein-AM staining of BMDMs. Always dispose the used stack of tissue papers into the corresponding safety waste.

5. Add $300 \mu \mathrm{l}$ Calcein-AM staining solution/well with a motorized single-channel pipet and incubate BMDMs at $37^{\circ} \mathrm{C}, 5 \% \mathrm{CO}_{2}$ for $15 \mathrm{~min}$.

6. Remove the Calcein-AM staining solution by quickly tilting the 24-well plate onto a fresh stack of tissue papers.

7. Wash BMDMs $3 x$ with $300 \mu l$ pre-warmed PBS and remove the wash solution by quickly tilting the 24-well plate onto the used stack of tissue papers.

8. Add $300 \mu \mathrm{l} \mathrm{PBS}$ to BMDMs of the untreated condition and $300 \mu \mathrm{l} \mathrm{FeHQ}$ solution to BMDMs of the FeHQ-treated condition with a motorized single-channel pipet and incubate BMDMs at $37^{\circ} \mathrm{C}, 5 \% \mathrm{CO}_{2}$ for $30 \mathrm{~min}$.

Note: BMDM incubation in FeHQ solution longer than 30 min will detrimentally affect BMDM membrane integrity. Therefore, subsequent BMDM harvest and flow cytometry analysis should also be performed as soon as possible.

9. Remove the solutions by quickly tilting the 24-well plate onto a fresh stack of tissue papers.

10. Wash BMDMs $3 x$ with $300 \mu l$ pre-warmed PBS and remove the wash solution by quickly tilting the 24-well plate onto the used stack of tissue papers

11. Add $250 \mu \mathrm{l}$ trypsin solution per 24-well with a motorized single-channel pipet and incubate BMDMs at $37{ }^{\circ} \mathrm{C}, 5 \% \mathrm{CO}_{2}$ for $5 \mathrm{~min}$.

12. Place 24-well plate on ice and add $750 \mu$ ice-cold complete DMEM to the wells in order to prevent further trypsinization.

13. Detach BMDMs by pipetting up and down with a $1,000 \mu \mathrm{l}$ single-channel pipet, transfer the cell suspension of every technical replicate to a separate $1.5 \mathrm{ml}$ reaction tube and store on ice in the dark.

14. After harvesting all BMDMs, centrifuge cell suspensions at $400 \times \mathrm{g}, 4^{\circ} \mathrm{C}$ for $5 \mathrm{~min}$.

15. Aspirate medium, resuspend BMDM pellet in $300 \mu \mathrm{l}$ FACS buffer and transfer to $5 \mathrm{ml}$ round-bottom FACS tubes.

16. Process all BMDM samples and store FACS tube on ice in the dark.

17. Analyze BMDM samples on a flow cytometer equipped with appropriate lasers and filters for the detection of Calcein fluorescence with excitation maximum $\sim 490 \mathrm{~nm}$ and emission maximum $\sim 510 \mathrm{~nm}$ (Sabnis, 2010) as soon as possible. 


\section{Data analysis}

1. Using appropriate flow cytometry analysis software, display the samples on a two-dimensional dot plot of FSC-A vs. SSC-A and gate on the BMDM population (Figure 3A).

2. For doublet discrimination, gate on single cells on an FSC-A vs. FSC-H dot plot (Figure 3A).

3. By measuring the unstained control sample, define unstained and Calcein ${ }^{+}$cells on a Calcein histogram (Figure 3A).

4. Analyze all BMDM samples by recording the Calcein fluorescence.

5. Determine the MFI (Calcein) (median fluorescence intensity) of Calcein ${ }^{+}$BMDMs.

6. Calculate the QIP as difference in MFI (Calcein) between untreated and FeHQ-treated BMDMs per every experimental condition:

$$
\text { QIP = MFI(untreated cells) }- \text { MFI(FeHQ-treated cells) }
$$

7. Visualize QIPs of BMDMs at different conditions in a column graph and perform statistical analysis (Figure 3B).

A
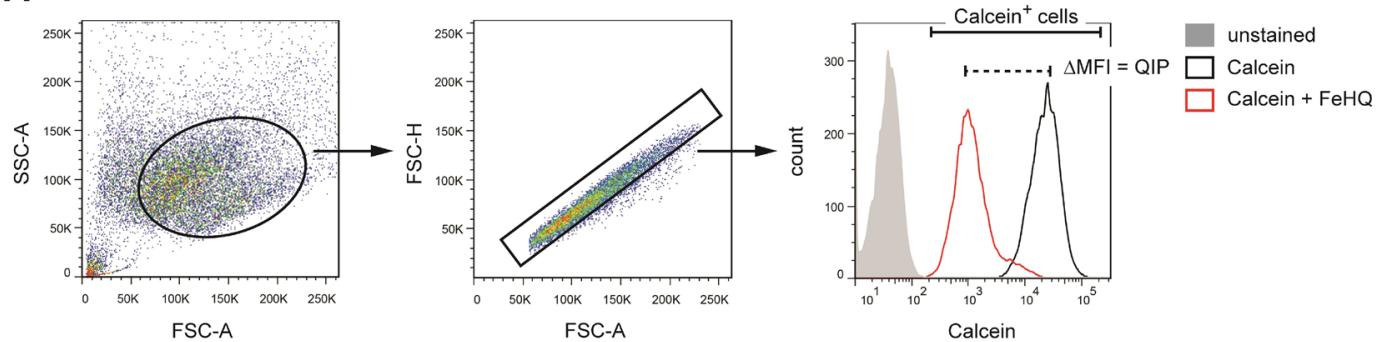

B

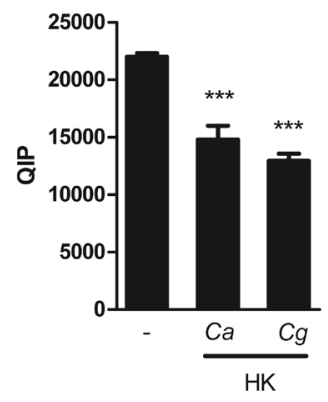

Figure 3. QIP analysis in BMDMs upon infectious stimuli. A. Gating strategy for QIP analysis. After doublet discrimination on an FSC-A vs. FSC-H dot plot, the MFI (Calcein) of Calcein $^{+}$cells is recorded. $\mathrm{B}$. BMDMs were infected with a $\mathrm{MOI}=5$ (pathogen:host cell ratio $=$ 5:1) of heat-killed $\left(70^{\circ} \mathrm{C}\right.$ for $10 \mathrm{~min}$ ) Candida albicans (Ca) or Candida glabrata $(\mathrm{Cg})$ for $12 \mathrm{~h}$ and subsequently analyzed for QIP alterations. Thereby, BMDMs upregulated their cytoplasmic iron levels during challenge with Candida spp., owing to the reduced QIPs upon infectious stimuli. MFI, median fluorescence intensity $\left({ }^{* * *} P\right.$-value $\left.<0.001\right)$. 


\section{$\underline{\text { Notes }}$}

1. The FeHQ solution should be adjusted approximately 30 min before BMDM incubation with Calcein-AM staining solution and should be mixed several times in between to ensure adequate complex formation of $\mathrm{Fe}^{2+}$ ions and 8-hydroxyquinoline.

2. As mentioned before, the Calcein-AM staining solution should be freshly prepared directly before the end of BMDM stimulation and immediately be used for subsequent BMDM staining owing to the high hydrolysis susceptibility of Calcein-AM in aqueous solutions. For example, $10 \mathrm{~min}$ in aqueous buffers decreases the BMDM staining efficiency of Calcein-AM for approximately $50 \%$.

3. Harvested BMDM samples should be analyzed by flow cytometry as soon as possible, since FeHQ loading of BMDMs shows adverse effects on cell membrane integrity upon prolonged storage of already processed samples.

4. This assay is highly accurate and the observed biological effects in BMDMs can be well repeated. However, the general amplitude of QIPs can vary between independent biological experiments, since the QIP is a function of Calcein fluorescence and, therefore, depends on the Calcein-AM staining efficiency of BMDMs.

5. For iron uptake studies into the cytoplasm, BMDMs should only be treated with solutions, for which the iron-containing chemicals have been diluted in ultra-pure, high quality water with minimal trace metal concentrations (e.g., grades suitable for trace element analysis). Otherwise, $\mathrm{Fe}^{2+} / \mathrm{Fe}^{3+}$ will form water-insoluble complexes in phosphate-buffered solutions and, therefore, will distort iron uptake by BMDMs.

6. This protocol can also be applied for other immune cell types in vitro and in vivo. Thereby, aliquots of $1 \times 10^{6}$ from the desired cell type (e.g., non-adherent cells or in vivo cell populations) should be transferred into $1.5 \mathrm{ml}$ reaction tubes and incubated with $200 \mu \mathrm{l}$ Calcein-AM staining solution respectively $\mathrm{FeHQ}$ solution at $37^{\circ} \mathrm{C}$ in a bench top thermomixer without agitation in the dark. The number of PBS washing steps in between can be reduced. However, in a pilot experiment, the required final Calcein-AM concentration, FeHQ composition and the incubation time for FeHQ loading should be optimized in detail for every cell type of interest via titration.

\section{$\underline{\text { Recipes }}$}

1. Complete DMEM

a. Add $10 \%$ heat-inactivated fetal calf serum (hiFCS) and $1 \times$ Penicillin/Streptomycin to DMEM

b. Store at $4{ }^{\circ} \mathrm{C}$ for up to one month

Note: For heat inactivation, incubate thawed FCS in a water bath at $56{ }^{\circ} \mathrm{C}$ for $30 \mathrm{~min}$ and invert FCS bottle every $10 \mathrm{~min}$. hiFCS can be stored in aliquots at $-20^{\circ} \mathrm{C}$ for up to 6 months.

2. L929-conditioned medium 


\section{Preparation of L929-conditioned medium}

Note: The following protocol for preparation of L929-conditioned medium was described previously (Bourgeois et al., 2009).

a. Cultivate M-CSF-producing L929 cells in $12 \mathrm{ml}$ complete DMEM and $10 \mathrm{~cm}$ round tissue culture dishes until confluency at $37^{\circ} \mathrm{C}, 5 \% \mathrm{CO}_{2}$

b. Harvest L929 cells from one $10 \mathrm{~cm}$ round tissue culture dish by gently scraping with a natural-rubber scraper and transfer the cell suspension into one T-150 cell culture flask with $80 \mathrm{ml}$ complete DMEM

c. Incubate $\mathrm{L} 929$ cells for 3 days until confluency at $37^{\circ} \mathrm{C}, 5 \% \mathrm{CO}_{2}$

d. Aspirate the medium and add $100 \mathrm{ml}$ DMEM per every T-150 cell culture flask Note: At this step, it is crucial to use DMEM (without hiFCS) instead of complete DMEM (supplemented with hiFCS).

e. Incubate $\mathrm{L} 929$ cells for 10 days at $37^{\circ} \mathrm{C}, 5 \% \mathrm{CO}_{2}$

f. Harvest the supernatants and filter them through a sterile disposable filter unit

g. Store $50 \mathrm{ml}$ aliquots at $-20^{\circ} \mathrm{C}$ for up to 12 months

\section{Testing of L929-conditioned medium}

a. Cultivate murine bone marrow cells in $3 \mathrm{ml}$ complete DMEM with increasing concentrations of L929-conditioned medium (0-20\%) in 6-well plates and incubate for 8 days at $37{ }^{\circ} \mathrm{C}, 5 \%$ $\mathrm{CO}_{2}$

Note: L929-conditioned medium from previous, already tested batches (or commercially available recombinant $\mathrm{M}$-CSF) can be used as positive control for macrophage differentiation.

b. Harvest adherent BMDMs by trypsinization and determine cellular yield of BMDMs by using an automated cell counter

c. Incubate BMDMs with antibodies against CD16/32 and macrophage-specific cell surface markers (e.g., CD11b, F4/80) to verify macrophage differentiation by FACS analysis

Note: BMDM differentiation via cultivation of bone marrow cells in L929-conditioned medium yields $\sim 98 \% \mathrm{CD} 11 \mathrm{~b}^{+} \mathrm{F} 4 / 80^{+}$macrophages.

d. Deduce from cellular yield and FACS analysis the optimal concentration of L929-conditioned medium for BMDM differentiation

3. BMDM medium

a. Add $15 \%$ L929-conditioned medium to complete DMEM

b. Store at $4{ }^{\circ} \mathrm{C}$ for up to one month

4. Stock solution preparation

a. For Calcein-AM stocks, dissolve $50 \mu \mathrm{g}$ solid Calcein-AM in $50 \mu \mathrm{l}$ anhydrous DMSO to prepare a $1 \mathrm{mM}$ stock. Freeze aliquots in amber reaction tubes at $-20^{\circ} \mathrm{C}$ protected from light for up to 4 months. Avoid multiple freezing/thawing cycles 
b. For $\mathrm{FeCl}_{2}$ stocks, dissolve $\mathrm{FeCl}_{2}$ in ultra-pure, high quality water and freeze $1 \mathrm{mM}$ stocks at $-20^{\circ} \mathrm{C}$

c. For 8-hydroxyquinoline stocks, dissolve 8-hydroxyquinoline in anhydrous DMSO, prepare $200 \mu \mathrm{M}$ stocks by dilution with PBS and store aliquots at $-20^{\circ} \mathrm{C}$

5. Calcein-AM staining solution

a. Dilute $1 \mu \mathrm{l}$ of $1 \mathrm{mM}$ Calcein-AM stock (f.c. $1 \mu \mathrm{M}$ ) in $1 \mathrm{ml}$ PBS, vortex and store the solution in the dark until use

b. Immediately incubate BMDMs in freshly prepared Calcein-AM staining solution to ensure maximum BMDM staining efficiency

6. FeHQ solution

a. Add $5 \mu$ l of $1 \mathrm{mM} \mathrm{FeCl}_{2}$ (f.c. $5 \mu \mathrm{M}$ ) to $945 \mu \mathrm{PBS}$ and vortex

b. Subsequently, add $50 \mu \mathrm{l}$ of $200 \mu \mathrm{M}$ 8-hydroxyquinoline (f.c. $10 \mu \mathrm{M}$ ) and vortex

c. Until BMDM treatment, mix FeHQ solution every $10 \mathrm{~min}$ to ensure adequate complex formation of $\mathrm{Fe}^{2+}$ ions and 8-hydroxyquinoline

7. Trypsin solution

a. Add $500 \mu \mathrm{l}$ of $10 \mathrm{x}$ trypsin to $4.5 \mathrm{ml} \mathrm{PBS}$ and $100 \mu \mathrm{l}$ of $500 \mathrm{mM}$ EDTA $(\mathrm{pH}=8.0$ )

Note: For trypsinization in general, it is crucial to use $\mathrm{Ca}^{2+}$ and $\mathrm{Mg}^{2+}$ free $\mathrm{PBS}$.

b. Incubate trypsin solution in a water bath at $37^{\circ} \mathrm{C}$ until use

8. FACS buffer

a. Dissolve $0.5 \mathrm{~g} \mathrm{BSA}$ (f.c. 1\%) in $50 \mathrm{ml} \mathrm{PBS}$

b. Filter-sterilise and store FACS buffer at $4{ }^{\circ} \mathrm{C}$ for up to 1 month

\section{Acknowledgments}

This protocol was supported by the Austrian Science Fund project InnateFun (FWF-I3319-B22) and in part by the project FUNGITECT (HEALTH-F3-2013-602125) from the EC-FP7 program to KK. We would like to acknowledge the studies from Du et al. (2015) and Siegert et al. (2015) for establishing this elegant method.

\section{Competing interests}

The authors declared that no competing interests exist.

\section{$\underline{\text { References }}$}

1. Bourgeois, C., Majer, O., Frohner, I., and Kuchler, K. (2009). In vitro systems for studying the interaction of fungal pathogens with primary cells from the mammalian innate immune system. Methods Mol Biol 470: 125-139. 
Please cite this article as: Riedelberger and Kuchler, (2020). Analyzing the Quenchable Iron Pool in Murine Macrophages by Flow Cytometry,Bio-protocol

2. Cabantchik, Z. I. (2014). Labile iron in cells and body fluids: physiology, pathology, and pharmacology. Front Pharmacol 5: 45.

3. Chobot, V., Hadacek, F., Bachmann, G., Weckwerth, W. and Kubicova, L. (2018). Antioxidant properties and the formation of iron coordination complexes of 8-hydroxyquinoline. Int J Mol Sci 19(12).

4. Du, J., Wagner, B. A., Buettner, G. R. and Cullen, J. J. (2015). Role of labile iron in the toxicity of pharmacological ascorbate. Free Radic Biol Med 84: 289-295.

5. Epsztejn, S., Kakhlon, O., Glickstein, H., Breuer, W. and Cabantchik, I. (1997). Fluorescence analysis of the labile iron pool of mammalian cells. Anal Biochem 248(1): 31-40.

6. Ganz, T. and Nemeth, E. (2015). Iron homeostasis in host defence and inflammation. Nat Rev Immunol 15(8): 500-510.

7. Ma, Y., Abbate, V. and Hider, R. C. (2015). Iron-sensitive fluorescent probes: monitoring intracellular iron pools. Metallomics 7(2): 212-222.

8. Nairz, M., Theurl, I., Swirski, F.K., and Weiss, G. (2017). "Pumping iron"-how macrophages handle iron at the systemic, microenvironmental, and cellular levels. Pflugers Arch 469: 397-418.

9. Prachayasittikul, V., Prachayasittikul, S., Ruchirawat, S., and Prachayasittikul, V. (2013). 8-Hydroxyquinolines: a review of their metal chelating properties and medicinal applications. Drug Des Devel Ther 7: 1157-1178.

10. Prus, E., and Fibach, E. (2008). Flow cytometry measurement of the labile iron pool in human hematopoietic cells. Cytometry A 73(1): 22-27.

11. Sabnis, R. (2010). Handbook of biological dyes and stains: synthesis and industrial applications Wiley Inc. USA. ISBN: 978-0-470-40753-0.

12. Siegert, I., Schodel, J., Nairz, M., Schatz, V., Dettmer, K., Dick, C., Kalucka, J., Franke, K., Ehrenschwender, M., Schley, G., Beneke, A., Sutter, J., Moll, M., Hellerbrand, C., Wielockx, B., Katschinski, D. M., Lang, R., Galy, B., Hentze, M. W., Koivunen, P., Oefner, P. J., Bogdan, C., Weiss, G., Willam, C. and Jantsch, J. (2015). Ferritin-mediated iron sequestration stabilizes hypoxia-inducible factor-1 $\alpha$ upon LPS activation in the presence of ample oxygen. Cell Rep 13(10): 2048-2055.

13. Soares, M. P. and Hamza, I. (2016). Macrophages and iron metabolism. Immunity 44(3): 492-504. 